\title{
TIME RESOLVED SPECTROSCOPY OF CATACLYSMIC VARIABLES: SS CYGNI
}

\author{
R. J. Stover, E. L. Robinson, R. E. Nather, and T. J. Montemayor \\ McDonald Observatory and Department of Astronomy, The University of Texas at Austin \\ Received 1980 January 25; accepted 1980 March 5
}

\begin{abstract}
Spectroscopic observations of radial velocity variations in the dwarf nova SS Cygni are presented, and the observing and analysis techniques are discussed in detail. Our analysis leads to the following orbital elements: $K_{\mathrm{R}}=153 \mathrm{~km} \mathrm{~s}^{-1}, K_{\mathrm{B}}=90 \mathrm{~km} \mathrm{~s}^{-1}, P_{\mathrm{ORB}}=0.275129$ days. Both $K_{\mathrm{R}}$ and $P_{\mathrm{ORB}}$ are significantly different from previously published measurements. We conclude that the white dwarf in SS Cygni may have a mass very near the Chandrasekhar limit.

Subject headings: stars: binaries - stars: dwarf novae - stars: individual
\end{abstract}

\section{INTRODUCTION}

Cataclysmic variables can be divided into four main groups: novae, dwarf novae, recurrent novae, and novalike variables. All four groups, while differing in detail, are unified under a single model of a close binary system in which the primary star is a white dwarf, and the secondary star fills its Roche lobe and is losing mass to the white dwarf through the inner Lagrangian point (Robinson 1976). Spectroscopically, cataclysmic variables can be very complicated. In many systems the major source of luminosity is the flattened disk of material (the accretion disk) that forms around the white dwarf. The disk produces a strong continuum along with Doppler-broadened emission lines of hydrogen, helium, and calcium. In addition, the stream of transferring material impacts onto the outer edge of the disk, producing a bright spot which can contribute significantly to both the continuum and line emission. Furthermore, in systems with orbital periods longer than about $5 \frac{1}{2}$ hours, the lobe-filling secondary star becomes bright enough so that a weak absorption spectrum begins to appear. All of these contributions to the spectrum can show rapid and long-term modulations in both intensity and radial velocity as the system rotates in its binary orbit. Old novae, in addition, may show a complex set of stationary emission lines arising from an expanding shell of ejected gas as well as high excitation lines arising in the accretion disk and bright spot. In order to separate all of these contributions and to study all of the periodic and random spectroscopic variations, a continuous series of short observations must be made as the two stars travel around their respective orbits. The observations must be able to properly record both very strong emission lines and very weak absorption lines in the same exposure. The Cassegrain Digicon spectrograph (CDS) on the $2.1 \mathrm{~m}$ Struve reflector allows us to obtain this kind of data, and for the last two years we have used it in a new program of timeresolved spectroscopy of cataclysmic variables. In this paper, the first in a series resulting from our new program, we describe the results of our study of the dwarf nova SS Cygni.

At minimum light SS Cyg has $m_{v} \approx 12$, making it the brightest member of the dwarf nova class of cataclysmic variables. Because of its relative brightness, SS Cyg has been extensively studied since its discovery in 1896, with spectroscopic observations dating back to 1921. The first extensive study of the radial velocity variations of SS Cyg at minimum light was done by Joy (1956), who showed that the system is one of the few double-lined spectroscopic binaries among cataclysmic variables and that it has an orbital period of about 6.5 hours. He found the spectrum to consist of broad, strong emission lines of hydrogen and $\mathrm{Ca}$ II along with a heavily veiled G5 absorption spectrum. The great width $(\sim 20 \AA)$ of the emission lines and the heavy veiling of the absorption lines made the radial velocity measurements exceedingly difficult with a resulting large scatter in the measurements. Unfortunately, the system does not eclipse, so that its orbital inclination cannot be measured directly; therefore direct mass determinations are not possible. When Walker and Chincarini (1968) made some additional radial velocity measurements they found that they had to introduce a $(d P / d T)$ term into Joy's ephemeris in order to properly phase their velocities with the earlier results. The validity of the resulting ephemeris was questioned by Smak (1969) and only partially supported by an extensive set of radial velocities obtained by Walker and Reagan (1971). A formal solution for this last set of data has never appeared in the literature.

The most recent radial velocity study of SS Cyg was done by Kiplinger (1979), who determined a greatly improved value for the $K$ velocity of the emission-line component. Using the model described earlier, he derived estimates for the masses of the system components. Unfortunately, Kiplinger was not able to measure the absorption line radial velocity variations reliably because of excessive veiling. 
In the current study we present new spectroscopic observations of SS Cyg at minimum light level. Because the observations are obtained with a linear response detector with wide dynamic range and because the data are recorded in a digital format, we have been able to apply analytic techniques which have greatly improved the radial velocity measurements. In $\S$ II we present the observations and briefly describe the observational techniques, and in § III we describe the analysis techniques and derive new estimates for the orbital elements.

\section{OBSERVATIONS}

\section{a) Instrumentation}

Because this is the first presentation of data from our new program, we will describe our instrumentation and our observational techniques in some detail. The basic design of the CDS and of the self-scanned Digicon detector has been described by Tull, Vogt, and Kelton (1979). The unique feature of the CDS is the Digicon detector which is a single-stage magnetically focused image tube, employing a cooled Reticon selfscanned photodiode array as the photoelectron target. The photodiode array is organized as two independent parallel linear arrays, each of 936 elements on $30 \mu \mathrm{m}$ centers. The detector is operated in the charge-storage mode, meaning that photoelectron signal is integrated in the arrays for a relatively long period of time, ranging typically from $\sim 1$ second to 60 seconds for stellar objects with $m_{v}$ between 10 and 15 . After the integration period, each array is read-out by sequentially measuring the integrated signal in each diode. Operated in this mode, we easily obtain photon shotnoise limited performance and an instantaneous dynamic range greater than 1000:1.

When observing stellar objects, one array is used to obtain the stellar spectrum while the other array is used to obtain the spectrum of the night sky. The entrance apertures for the two arrays are defined by a pair of circular holes in a metal mask located just above a common entrance slit. The stellar image is centered in one of the entrance apertures by adjusting the tilt of a rectangular quartz prism, known as the wobble block, which is located a few centimeters above the entrance slit. Typically six to eight integrations are obtained with the stellar image on one of the apertures, after which the image is switched to the other aperture by rotating the wobble block. Operated in this manner, the sky observation follows the stellar observation for one array and precedes the stellar observations for the other array.

The sequence of six to eight integrations per array is repeated until the observer terminates the observations. Each observation, therefore, produces two independent, time-interlaced, sky-subtracted spectra of nearly identical characteristics.

\section{b) Computer Control}

The CDS design continues the McDonald philosophy of software-dominated data collection instruments. This means that the hardware associated with the CDS is relatively simple and is under direct control of a computer, in this case a Data General Corporation Nova 2/10. Proper sequencing of events is maintained by the computer software rather than by complex hard-wired controllers. In addition, the raw digital data are entered directly into the computer memory, thus allowing initial signal processing before the data are recorded in final form. Another consequence of software-dominance is that the operating characteristics, the ease of operation, and the research flexibility of the instrument are determined largely by the data collection program. Therefore, we have invested a large fraction of our initial efforts in the development of a versatile data collection program capable of efficiently performing high-speed time-resolved spectroscopy. The CDS can be controlled directly with simple console commands and manipulation of the computer's front panel switch inputs or by use of a control macro. Macros, written in a high-level language designed specifically for the CDS, give the observer fully automatic control over the instrument and the data collection sequence, and are the key to doing high-speed spectroscopy.

Most of our macros execute a cyclic observing sequence. For instance, a typical macro might obtain a single 100 second exposure of the wavelength calibration lamps followed by four 5 minute exposures of a cataclysmic variable. The sequence would be repeated by the macro until the observer instructed the computer to stop. The macro would then finish the current exposure and, if the current exposure was not of the wavelength calibration lamp, a final calibration spectrum would be obtained. Integration timing, wobble block position, summation of integrations into an exposure, sky subtraction, exposure timing, calibration lamp control, and data recording are all automatically sequenced by the program, thus freeing the observer to monitor the progress of the observation and to make adjustments in the observing based on its progress.

\section{c) Data}

The observations of SS Cyg consist of 35 pairs of spectra obtained with the CDS on the $2.1 \mathrm{~m}$ Struve reflector at McDonald Observatory on the nights of 1978 July 10 through 1978 July 14 UT and on 1978 August 16 UT. The star was observed through a 1" entrance aperture at a dispersion of approximately $1.9 \AA$ per pixel with each spectrum covering a useful wavelength range of $3700 \AA-5000 \AA$. The data collection macro described in $\S$ II $b$ was used except that a single 20 minute exposure of SS Cyg was obtained between each wavelength calibration spectrum. Note that the total exposure of 20 minutes is divided between the two diode arrays in an interlaced fashion so that the 
individual spectra consist of 10 minutes on SS Cyg and 10 minutes on the night sky. The two observations on August 16 were slightly different from the other observations because they were "micro-scanned," a process in which an additional pair of spectra are produced by using the image tube's magnetic deflection coils to shift the spectrum by one-half the width of the diode, thereby increasing the sampling density to two samples per diode width, with a resulting increase in effective resolution. In this case, a single observation provides two pairs of spectra, each pair consisting of an undeflected spectrum and a deflected spectrum from one of the diode arrays. In addition, the exposure per spectrum is decreased by an additional factor of 2 , to 5 minutes per spectrum for a total exposure of 20 minutes.

In addition to the SS Cyg spectra, each night included observations of the flux standard star HR 8553 and a tungsten filament lamp in order to determine the instrument response.

\section{ANALYSIS}

\section{a) Calibrations}

To obtain the best possible radial velocities, the spectra to be measured must have excellent wavelength calibrations; and to obtain the best possible wavelength calibrations, the spectra must have reasonably good flux calibrations. We therefore begin the reductions by deriving the instrumental response from nightly observations of the flux standard star HR 8553 and a tungsten filament lamp. The observations of HR 8553 give us a measure of the slowly varying component of the response function which arises primarily from the change in sensitivity of the Digicon photocathode as a function of wavelength. The intrinsically smooth spectrum of the lamp gives us a measure of the component of the response function which varies with a high spatial frequency. The principal high-frequency variations are two- and four-point periodic variations of from $1 \%$ to $5 \%$ in the sensitivity introduced by the analog signal-processing electronics. Removal of these variations is especially important because most of the absorption lines seen in SS Cyg have essentially the same amplitude and spatial frequencies and can therefore suffer severe distortion. These periodic variations can be removed with high accuracy because the amplitude of the variations remains very stable. Virtually noise-free lamp spectra can be obtained quickly and easily with the Digicon, which enjoys both a wide dynamic operating range and freedom from phosphor afterflow.

The typical response function is shown in Figure 1 in the sense that a spectrum multiplied by the response function produces an instrument-free relative flux, $f_{v}$. The response function is usually accurate to about 5$10 \%$ overall, which is sufficiently accurate to calibrate the spectra of SS Cyg that can suffer from additional systematic flux losses at the narrow entrance slit.

Argon and neon discharge lamps are observed simultaneously to obtain the wavelength calibration spectra which bracket every SS Cyg observation. Before the wavelength calibration spectra are measured they are multiplied by the derived response function. Typically, 15 unblended argon or neon lines are algorithmically identified in each spectrum. The centers of the lines are accurately determined by fitting a Gaussian function to each line. A fifth-order polynomial is then fit by least squares to the line-centers to give position in the spectrum as a function of wavelength.

Figure 2 shows how the wavelength calibration changed as a function of time during the night of July 14. The primary source of wavelength change is mechanical flexure in the spectrograph. Because the flexure produces a smoothly varying change in wavelength as a function of time, we have been able to remove its effects and obtain accurate wavelength calibrations for the SS Cyg spectra by linearly interpolating the bracketing wavelength calibrations to the time of midexposure for each observation of SS Cyg.

The final step in the calibration procedure is to multiply each SS Cyg observation by the response function and to apply a first-order correction for atmospheric extinction.

\section{b) Absorption-Line Radial Velocities}

\section{i) Methods}

Up to the present, most radial velocity studies of cataclysmic variables, including SS Cyg, have been done photographically. This has presented especially difficult problems in the measurement of the absorption-line radial velocities because the visible lines are extremely faint and very few in number. Walker and Chincarini (1968), for instance, used only Fe I $\lambda \lambda 4132$ and 4143. Kiplinger (1979), who had only Ca I $\lambda \lambda 6122$ and 6162 to work with, rejected his own absorption line velocity measurements because the lines were so heavily veiled. But when SS Cyg is observed electronically, as with the Digicon, our ability to measure very faint lines is greatly improved, radically increasing the number of lines available for measurement. In fact, as will be shown in Figure 4, we now have the problem of extracting velocities from a very large number of lines most of which, at Cassegrain dispersion, are heavily blended.

The most effective means of solving this measurement problem is to cross-correlate the absorption-line spectrum of SS Cyg with the spectrum of a velocity standard star of similar spectral type. This procedure has been applied successfully to stars at coudé dispersions (Griffin 1967) and to galaxies at Cassegrain dispersions (Tull, Vogt, and Kelton 1979), and in this paper we apply it to measuring the velocity variations of the absorption line spectrum of SS Cyg.

\section{ii) Transformations}

In order to use the cross-correlation technique to measure the wavelength displacement arising from the 


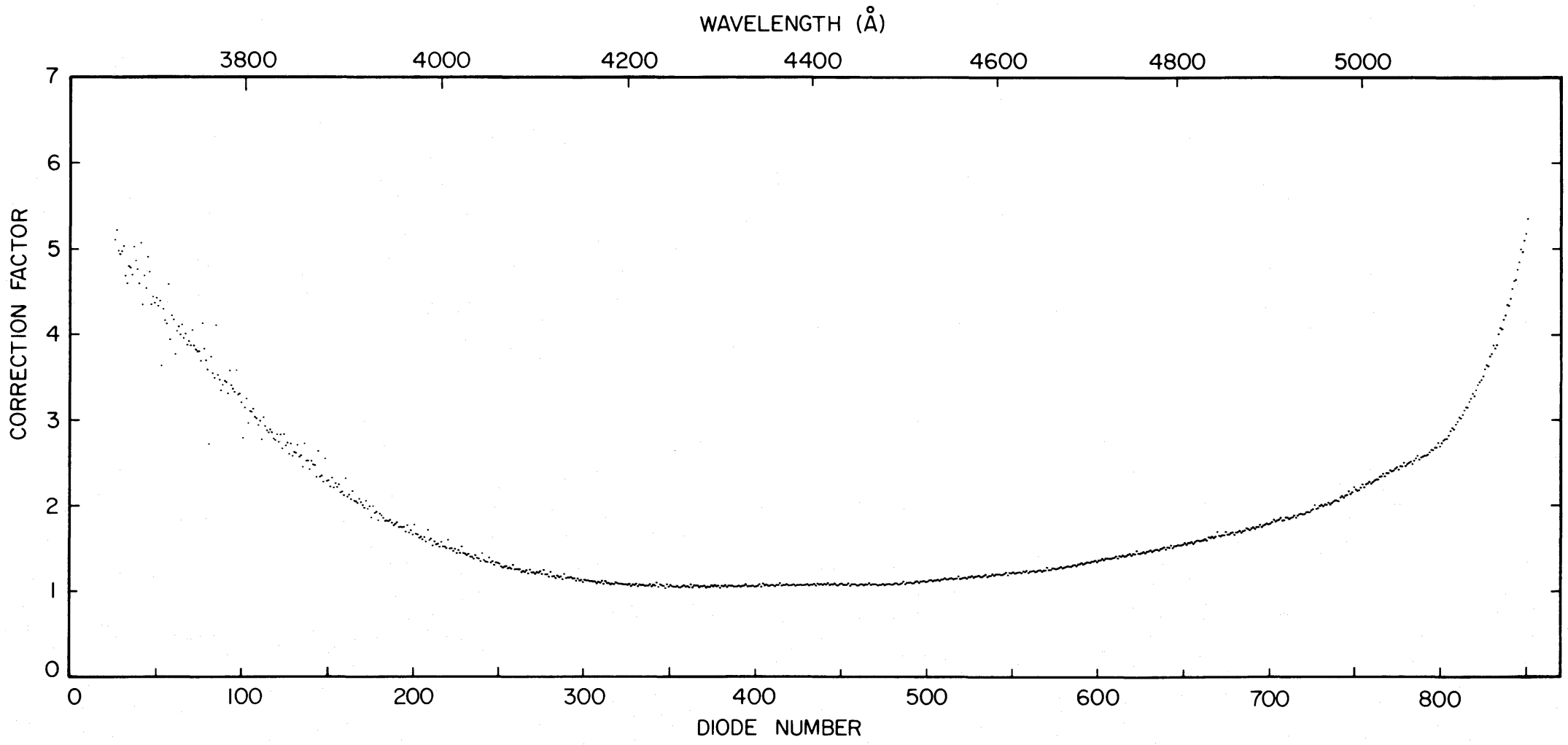

FIG. 1. - Response function for the CDS. Multiplication by the response function produces an instrument-free relative flux, $f_{v}$. 


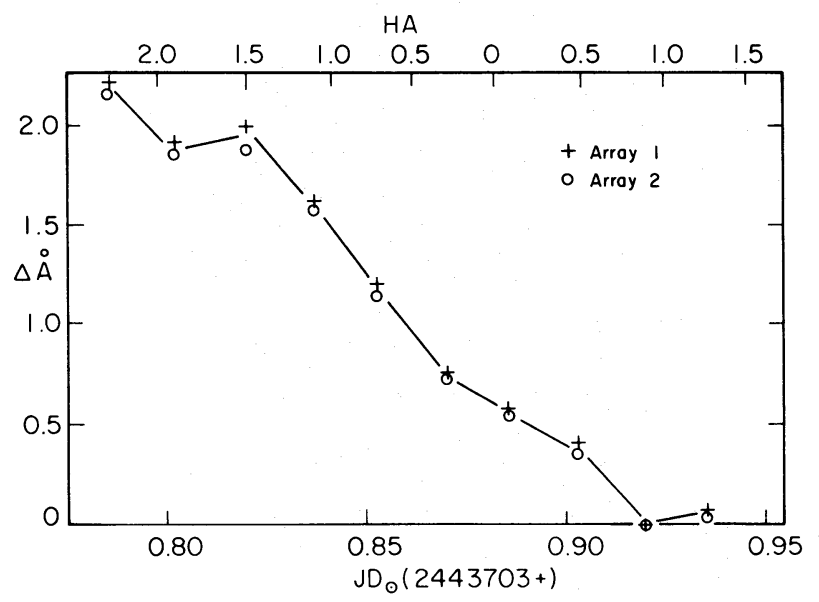

FIG. 2.-Instrumental change in wavelength as a function of time for observations on the night of 1978 July 14, UT.

Doppler effect the displacement must be uniform, or nearly so, throughout the spectrum. At coudé dispersions this condition is nearly satisfied because a very small wavelength range is usually covered. However, at our Cassegrain dispersions, we cover wavelengths from $3700 \AA$ to $5000 \AA$ in a single spectrum, so that the wavelength displacement varies by as much as $35 \%$. We therefore transform our spectra onto a logarithmic or velocity scale in which the apparent displacement is independent of wavelength and is a function only of the radial velocity. The transformed spectra have a wavelength scale such that

$$
\log \lambda=A+B x \text {, }
$$

where $x$ is the pixel number and where $A$ and $B$ are constants. An apparent radial velocity, $V$, is then represented by a uniform displacement, $\Delta x$, of the entire spectrum and is given by

$$
V=c\left[e^{B \Delta x}-1\right] \text {, }
$$

where $c$ is the speed of light.

In the context of a cross-correlation, $\Delta x$ is the relative displacement required to bring a comparison spectrum into registration with the measured spectrum, and $V$ is the relative radial velocity. The transformation of our spectra onto a logarithmic scale necessarily involves interpolation of the data. But all simple interpolation schemes tend to filter the data at high frequencies, with a corresponding reduction in effective spectral resolution. This unfortunate characteristic of most interpolation schemes becomes especially acute for the absorption line spectra, because essentially all of the lines are very narrow and unresolved. Therefore any degradation in the effective spectral resolution would directly degrade our ability to measure radial velocity variations. We have therefore developed a sine function interpolation procedure which preserves the full spectral resolution. Since the technique has wide general application when dealing with the transformation or interpolation of digital spectra we give a full description of our procedure in Appendix A.

The final step required before a cross-correlation can be done is to remove the continuum. Because we have already removed the instrumental response from our data, the continuum slope is fairly uniform, and practically any broad, low-frequency filter can be used. In fact, we have found that the measured radial velocities are insensitive to either the width or shape of the filter function as long as it does not significantly affect the high frequencies.

\section{iii) Procedure}

The absorption line radial velocities presented here were obtained using HD 198550 as the comparison star. This star was chosen from among a sample of 15 G4-K5 stars observed in 1978 August because its spectrum closely matches the spectrum of the secondary star in SS Cyg. We find, however, that the choice of comparison star is not critical. If the comparison star is within about three-tenths of a spectral type of the star being measured, the resulting velocity measurements are not affected by the mismatch in spectra, and for greater differences in spectral types the accuracy of the velocity measurements slowly decreases, but without the introduction of systematic errors. The comparison star spectrum is prepared by transforming it onto a velocity scale and by removing the continuum, as described in the previous section. In addition, we mask out the regions around $\mathrm{H} \beta, \mathrm{H} \gamma$, and $\mathrm{H} \delta$ as well as the remainder of the spectrum blueward of $\mathrm{H} \epsilon$ since, in the SS Cyg spectra, these regions are dominated by the very strong emission lines of the accretion disk.

Each transformed and continuum-filtered spectrum of SS Cyg is then cross-correlated against the prepared comparison spectrum, giving us a series of points on the cross-correlation function. The position of the centroid of the function, which gives us the displacement $\Delta x$, is measured by fitting a Gaussian curve to the peak of the computed cross-correlation. A typical cross-correlation and the fitted Gaussian curve are shown in Figure 3.

\section{c) Emission Line Radial Velocities}

\section{i) Method}

The emission line radial velocity variations of $\mathrm{H} \beta$, $\mathbf{H} \gamma$, and $\mathbf{H} \delta$ were measured simply by fitting a symmetric polynomial to the emission-line wings. We fit only to the wings of the line profiles, which arise from high-velocity material close to the white dwarf, and exclude the line centers, which arise from the asymmetric outer regions of the disk and the bright impact spot. A similar technique applied to photographic spectra of $\mathrm{H} \alpha$ was used in the study of SS Cyg by Kiplinger (1979) and in the study of EM Cyg by Robinson (1974). We find, however, that measuring the wings on our original spectra give results which differ from line to line and that the differences are 


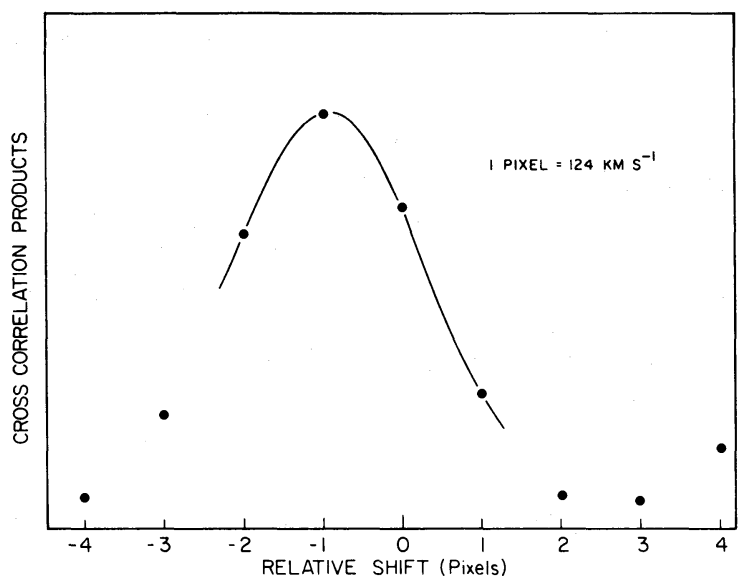

FIG. 3.-A typical absorption line cross-correlation function and the fitted Gaussian function. The solid curve is drawn between the points included in the fit. The centroid of the Gaussian curve gives the relative radial velocity.

caused by the effect of the multitude of underlying absorption lines of the secondary star on the emission line profiles. We have, therefore, taken full advantage of our linear-response digital data by removing the absorption line spectrum before the emission line radial velocities are measured.

\section{ii) Procedure}

Our procedure for removing the absorption lines from the emission line spectrum is relatively simple. We just use HD 198550 as an approximation to the true absorption line spectrum and subtract the approp- riate amount to minimize, in the sense of least-squares, the residual absorption lines in the SS Cyg spectra. For this technique to be successful the stellar spectrum must be a fairly close match to the secondary star of SS Cyg in both line strength and wavelength. HD 198550 meets the first requirement, and since it was used to determine the absorption line radial velocities, we know exactly how much to shift its spectrum to bring it into exact alignment with each of our SS Cyg spectra. Therefore, for each SS Cyg observation, we use our sinc function interpolation routine to produce a transformed spectrum of HD 198550 with the same apparent radial velocity. A very simple least-squares analysis then determines for each pair of spectra exactly how much of the HD 198550 spectrum to subtract to minimize the remaining absorption lines. The results of this procedure are illustrated in Figure 4. To show the absorption line spectrum with the highest possible signal-to-noise ratio we first transformed all of our SS Cyg spectra onto a logarithmic scale with the absorption line radial velocities removed. The spectra could then be co-added with no velocity smearing in the absorption lines. The result is shown in the upper half of the figure. This spectrum was then compared with all of our $\mathrm{G}$ - and $\mathrm{K}$-star spectra to determine the closest match. The lower half of the figure shows the same spectrum with HD 198550 subtracted. No smoothing has been applied to either spectrum. The effect on the continuum regions is dramatic, but more importantly, the emission lines become much more symmetrical and smooth. The improvement in $\mathbf{H} \gamma$ is particularly apparent where the severe distortion by the G-band of the late-type star has been removed.

Once the absorption spectrum has been removed we measure the centroid of the emission lines by fitting a

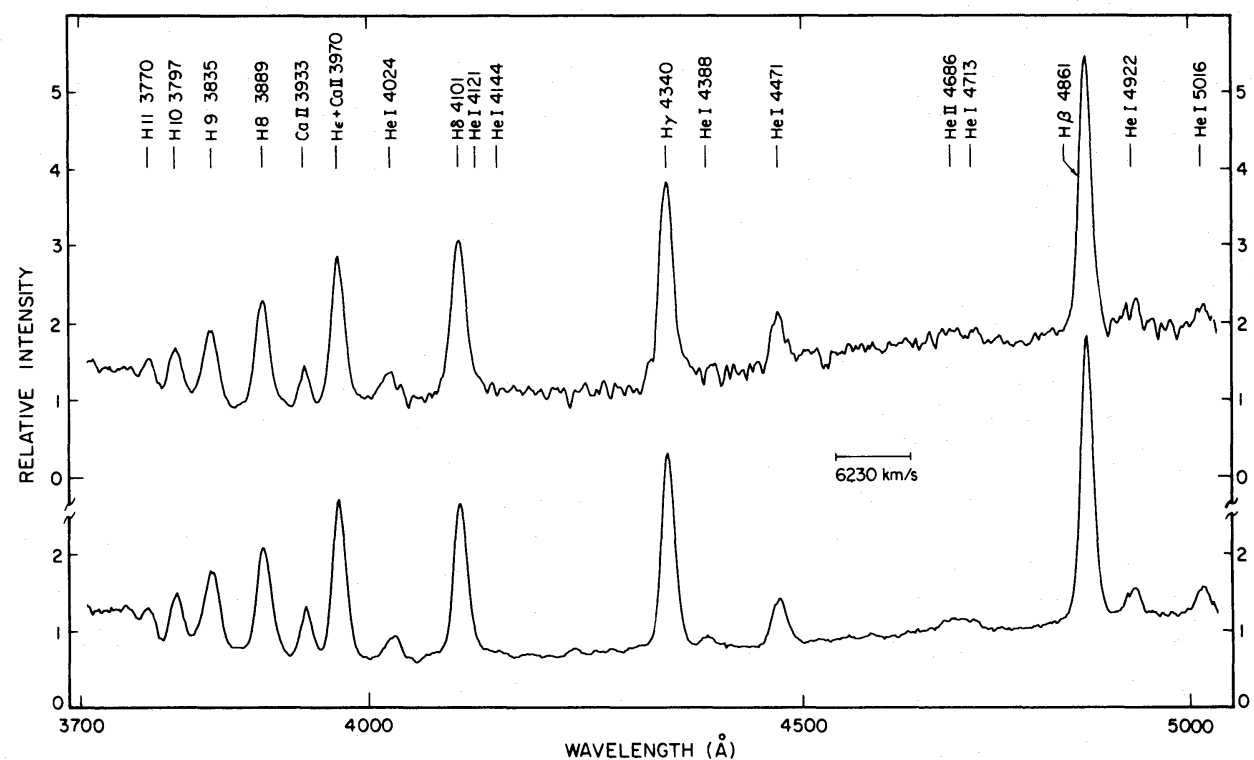

FIG. 4.- Removal of late-type component from the SS Cyg spectrum. Upper, the visual spectrum of SS Cyg; lower, the spectrum of SS Cyg after subtraction of the late-type star HD 198550. 


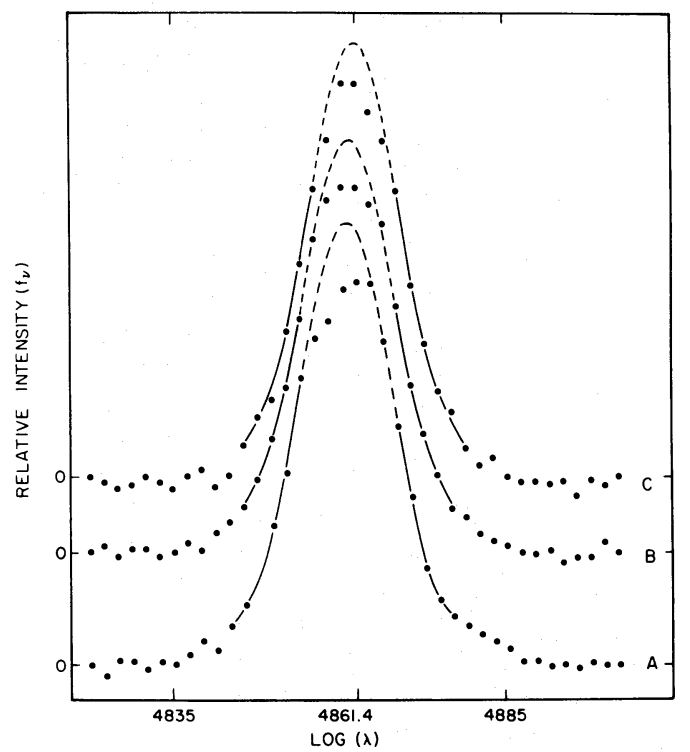

FIG. 5. $-\mathrm{H} \beta$ and the 6 th order polynomial fit. Three examples are shown to illustrate the varying distortions in the central region of the line.

symmetric polynomial to the wings using a leastsquares routine. We have found that the most reliable radial velocities are obtained by fitting a sixth-order polynomial of the form

$$
d=\sum_{i=0}^{3} a_{i}\left(x-x_{0}\right)^{2 i} .
$$

This polynomial has five fitting parameters: the four profile coefficients $a_{i}$, and the centroid $x_{0}$. Points in the wings were included to a velocity of about $1000 \mathrm{~km} \mathrm{~s}^{-1}$ from the line center. Beyond this velocity the slope of the line profile becomes relatively shallow and photon shot noise from the continuum becomes important so that little additional information about the line center can be obtained. The choice of which points to include in the fit near the line center was based on the visual appearance of the lines, with an attempt to avoid the effects of bright spot as much as possible. Three examples of the fit to $\mathrm{H} \beta$ are shown in Figure 5 where the polynomial curve is drawn as a solid line between the data points included in the fit. As illustrated by the three examples, the wings maintain good symmetry even though the central regions exhibit severe profile distortion. The detailed behavior of the emission line profiles and intensities will be discussed in a later paper.

\section{d) Results}

The measured heliocentric radial velocities for SS Cyg are presented in Table 1, where we have averaged the results for both diode arrays and for all three hydrogen emission lines. The second column of Table 1 gives the mean heliocentric Julian date for each observation. In Table 2 we present the orbital param-
TABLE 1

SS Cygni Heliocentric Radial Velocities

\begin{tabular}{|c|c|c|c|}
\hline No. & $\begin{array}{c}\mathrm{JD}_{\odot} \\
2443000 .+\end{array}$ & $\underset{\mathrm{km} \mathrm{s}^{-1}}{\mathrm{v}_{\mathrm{em}}}$ & $\begin{array}{c}v_{a b} \\
k m s^{-1}\end{array}$ \\
\hline 1 & 699.86509 & -36 & -19 \\
\hline 2 & 699.88169 & -5 & -49 \\
\hline 3 & 699.89829 & 27 & -85 \\
\hline 4 & 699.91490 & 70 & -151 \\
\hline 5 & 699.93150 & 65 & -167 \\
\hline 6 & 700.80243 & 45 & -126 \\
\hline 7 & 700.81904 & 27 & -53 \\
\hline 8 & 700.83566 & -2 & -9 \\
\hline 9 & 700.85227 & -38 & 45 \\
\hline 10 & 700.86889 & -69 & 98 \\
\hline 11 & 700.88550 & -115 & 126 \\
\hline 12 & 700.90212 & -118 & 130 \\
\hline 13 & 700.92052 & -109 & 126 \\
\hline 14 & 700.93713 & -77 & 99 \\
\hline 15 & 701.72240 & -112 & 130 \\
\hline 16 & 701.73900 & -109 & 124 \\
\hline 17 & 701.75560 & -86 & 106 \\
\hline 18 & 701.77221 & -66 & 59 \\
\hline 19 & 701.78881 & -59 & 13 \\
\hline 20 & 701.86997 & 65 & -165 \\
\hline 21 & 701.88657 & 62 & -152 \\
\hline 22 & 701.90318 & 56 & -113 \\
\hline 23 & 701.91978 & 23 & -62 \\
\hline 24 & 701.93638 & -4 & 3 \\
\hline 25 & 703.79597 & 74 & -172 \\
\hline 26 & 703.81367 & 69 & -147 \\
\hline 27 & 703.83027 & 53 & -110 \\
\hline 28 & 703.84687 & 34 & -55 \\
\hline 29 & 703.86348 & -11 & -6 \\
\hline 30 & 703.88008 & -27 & 60 \\
\hline 31 & 703.89668 & -67 & 104 \\
\hline 32 & 703.91329 & -93 & 134 \\
\hline 33 & 703.92989 & -102 & 138 \\
\hline 34 & 736.66480 & -98 & 136 \\
\hline 35 & 736.68343 & -80 & 138 \\
\hline
\end{tabular}

eters derived from the radial velocities of Table 1 , and in Figure 6 we show both the measured velocities and the calculated velocity curve folded on the orbital period.

Our initial analysis of the orbital elements gave a formal eccentricity of $0.01 \pm 0.01$ for the absorptionline radial velocity variations and $0.01 \pm 0.03$ for the emission-line radial velocity variations. Therefore, in deriving the orbital parameters of Table 2, we have assumed $e=0$ and have solved for only the amplitude and phase of the radial velocity variations. The absorption-line radial velocities are the most accurate, with an rms error of fit of about $7 \mathrm{~km} \mathrm{~s}^{-1}$, corresponding to about $1 / 20$ pixel in the original spectra. The emission line measurements are nearly as accurate with an rms error of fit of just over $9 \mathrm{~km} \mathrm{~s}^{-1}$.

The systemic $\gamma$ velocity of SS Cyg derived from the absorption-line spectra depend on the measured $\gamma$ velocity of the comparison star used in the crosscorrelation. HD 198550 has a measured radial velocity of $-13.3 \mathrm{~km} \mathrm{~s}^{-1}$ (Heard 1956), which results in a derived $\gamma$ velocity of $-16 \mathrm{~km} \mathrm{~s}^{-1}$ for SS Cyg. This is 
TABLE 2

SS Cygni Orbital Elements

\begin{tabular}{|c|c|c|}
\hline Element & $\begin{array}{c}\text { Emission } \\
\text { (White Dwarf) }\end{array}$ & $\begin{array}{c}\text { Absorption } \\
\text { (Late-type Star) }\end{array}$ \\
\hline \multicolumn{3}{|c|}{$P=0.275129 \pm 0.000001, e=0, \gamma=-15 \pm 4$} \\
\hline 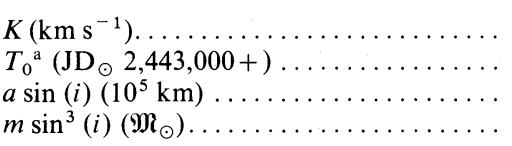 & $\begin{aligned} 90 & \pm 2 \\
699.9458 & \pm 0.0012 \\
3.40 & \pm 0.08 \\
0.26 & \pm 0.02\end{aligned}$ & $\begin{array}{c}153 \pm 2 \\
700.0764 \pm 0.0006 \\
5.79 \pm 0.08 \\
0.15 \pm 0.01\end{array}$ \\
\hline
\end{tabular}

${ }^{a} T_{0}=$ time of maximum positive velocity

not significantly different from the $-15 \mathrm{~km} \mathrm{~s}^{-1} \gamma$ velocity measured from the hydrogen emission lines. Furthermore, 11 of the $15 \mathrm{G} 4$ to $\mathrm{K} 5$ comparison stars observed in 1978 August have measured radial velocities, and the average $\gamma$ velocity of SS Cyg derived by cross-correlation against all 11 stars is $-15 \mathrm{~km} \mathrm{~s}^{-1}$. We have therefore adopted $-15 \mathrm{~km} \mathrm{~s}^{-1}$ as the $\gamma$ velocity for both the emission and absorption line radial velocity curves.

In Table 1 we averaged all available measurements for a single observation. However, because this is the first presentation of data from our new program, it is worth examining our results in more detail. In particular, we can obtain two independent measurements of the orbital elements because the Digicon detector produces two independent spectra for each observation. Furthermore, we can treat each of the three measured hydrogen emission lines separately for a total of six independent measurements of the white dwarf radial velocity variations. As can be seen in Table 3, where we list the six $\gamma$ and $\mathrm{K}$ velocities, the spectra from the two diode arrays produce essentially identical results. The semiamplitude of the velocity variation, $\mathrm{K}$, is reproduced very closely for all six measurements. It is interesting to note that before we removed the late-type absorption spectrum, differences in $\mathrm{K}$ as large as $40 \mathrm{~km} \mathrm{~s}^{-1}$ were seen between $\mathrm{H} \gamma$ and $\mathrm{H} \delta$. The only systematic difference apparent in the velocities of Table 3 is the $6 \mathrm{~km} \mathrm{~s}^{-1}$ difference between the $\gamma$ velocities derived from the two diode arrays. Such a small difference, if real, is quite acceptable at our Cassegrain dispersion and amounts to a relative position error between the two diode arrays of only $1.5 \mu \mathrm{m}$.

\section{DISCUSSION}

Several significant results are presented in Table 2 . The most important result is the new measurement of the semiamplitude $K$, of the late-type component radial velocity variation. Our measurement is significantly larger than Joy's value and demands a mass ratio of $\mu=1.70$. With such a large mass ratio, the white dwarf must be very close to the upper limit on white dwarf masses. Unfortunately the inclination of the system is not known so the masses of the components can not be computed from first principles. But if we assume that the secondary star has an internal structure similar to a main-sequence star, then we can

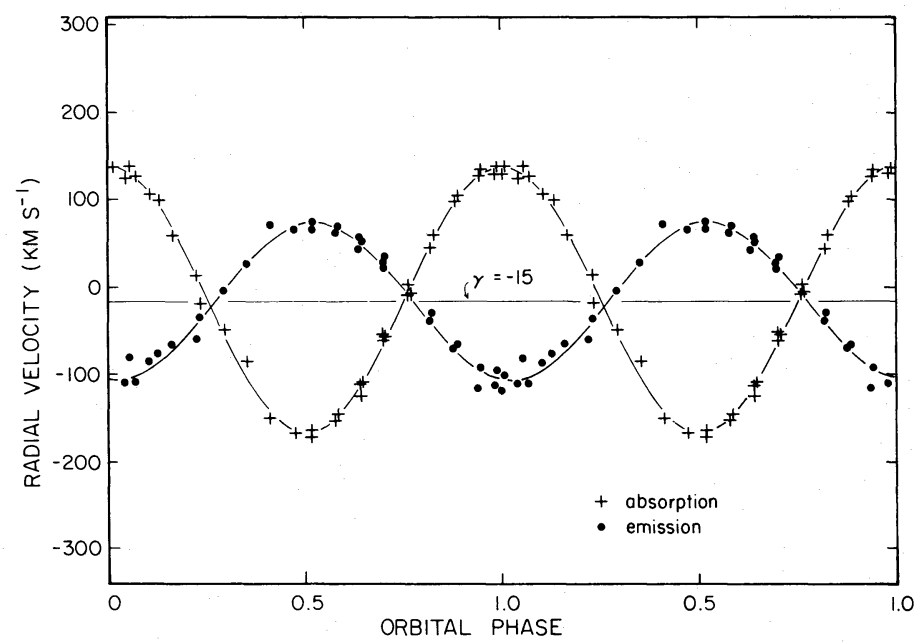

FIG. 6.-Measured radial velocities of SS Cyg. Emission-line velocities are represented by circles, absorption-line velocities by pluses. The solid curves correspond to the circular orbit solutions in Table 2. 
TABLE 3

Orbital ElEMENTS FOR INDIVIDUAl EMISSION LiNES

\begin{tabular}{cccc}
\hline Line & Array 1 & Array 2 & Average \\
\hline $\mathrm{H} \beta \ldots \ldots \ldots \ldots \ldots \ldots \ldots$ & -18 & -13 & -16 \\
$\mathrm{H} \gamma \ldots \ldots \ldots \ldots \ldots \ldots \ldots$ & +88 & +87 & +88 \\
$\mathrm{H} \delta \ldots \ldots \ldots \ldots \ldots \ldots \ldots$ & -21 & -11 & -16 \\
& +83 & +91 & +87 \\
Average $\ldots \ldots \ldots \ldots \ldots \ldots$ & -12 & -9 & -11 \\
& +93 & +91 & +92 \\
& +88 & -11 & $\ldots$ \\
\hline
\end{tabular}

use the same analysis as Kiplinger (1979) to derive an inclination of $35^{\circ}$, and mass estimates $\mathfrak{M}_{r}=0.78 \mathfrak{M}_{\odot}$ and $\mathfrak{M}_{d}=1.33 \mathfrak{M}_{\odot}$ for the secondary star and for the white dwarf, respectively.

A second result, important both observationally and theoretically, is that Joy's (1956) orbital period of 0.276244 days simply does not fit our data. Based on our radial velocities alone, which cover a 1 month baseline, we have derived an unambiguous period of 0.2751 days; if we phase our data with Kiplinger's (1979) data and Joy's data, we obtain a constant orbital period of 0.275129 days with good agreement in phase among all sets of data. Cowley (1980) reports that this period also agrees with her recent radial velocity observations of SS Cyg. While we do not rule out the possibility of a small $P$ term, the previous claim for a significant change in the orbital period by Walker and Chincarini (1968) appears to be unjustified since it was based on Joy's alias of the true orbital period.

A third significant result shown in Table 2 is an apparent $9^{\circ}$ phase shift between the velocity curve of the late-type star and of the accretion disk. The initial assumption was that the radial velocities determined from the emission lines of the accretion disk correspond to the actual velocities of the white dwarf. However, the apparent phase shift indicates that the disk may have a large-scale asymmetry in its emission distribution. We have deliberately avoided the central regions of the emission lines, which clearly show severe distortion arising from the changing aspect of the bright impact spot located on the edge of the disk. The $9^{\circ}$ shift, however, does not seem to be strongly related to the distortion near the center of the line but is rather representative of the entire line and therefore of the entire accretion disk.

Kiplinger (1979) reported a curious doubling of the absorption lines on several of his photographic spectra. We have searched for but have failed to find any evidence for such splitting in our data. Kiplinger also points out that variations in absorption-line strengths over the surface of the late component could result in systematic errors in the radial velocity measurements, presumably by displacing the apparent centroid of the star away from the true center of mass. Due to the changing aspect such a displacement would be expected to vary as the late component orbits the white dwarf, producing an apparent, but fictitious eccentricity. But since our absorption-line velocity measurements set an upper limit of 0.01 on the eccentricity, line-strength variations do not seem to be important in SS Cyg at minimum light.

\section{CONCLUSIONS}

The presentation of this work has served a twofold purpose: to describe our new program of timeresolved spectroscopy of cataclysmic variables and to present our first results on the dwarf nova SS Cyg. We chose SS Cyg for our initial investigation because it is the brightest and most thoroughly studied dwarf nova spectroscopically and would therefore provide a baseline against which we could judge our results. However, we quickly realized that the quality of our data exceeded that of all previously published work and that the measurements of some of the most fundamental physical parameters of SS Cyg were in serious error. We have found, for instance, that the orbital period is 1.6 minutes shorter than formerly believed, rendering all previous claims for a $(d P / d T)$ term invalid. In addition, we find a large mass ratio for the system which indicates that the white dwarf component must be very massive, and close the Chandrasekhar limit for helium-core objects. Continued accretion may, in time, cause it to exceed that limit, with results that will certainly be observationally and theoretically fascinating.

Our results on SS Cyg have shown that the high stability of the new CDS coupled with careful attention to calibration permits us to obtain short exposure radial velocity measurements on a cataclysmic variable with $m_{v}=12$ which are limited, at $1 / 20$ pixel, primarily by our Cassegrain dispersion. Furthermore, our experience indicates that observations with the same 20 minute exposure on similar systems 2 magnitudes fainter can produce velocity measurements nearly as good, with an expected mean error of about $15 \mathrm{~km} \mathrm{~s}^{-1}$. This means that a large number of faint cataclysmic variables are within the useful range of the CDS and, for the first time, are candidates for radial velocity study.

This work has been supported by the National Science Foundation through grants AST 76-23882 and AST 79-06340.

\section{APPENDIX}

In order to change the wavelength scale of our digital stellar spectra, we must interpolate between the sample points of the spectrum. If done without care, the interpolation can reduce the total information content of the 
spectrum. For example, a simple linear interpolation between the sample points will smooth the spectrum to a greater or lesser degree, and this smoothing removes information at scales near the sampling interval. Unresolved and underresolved features in the spectrum will be degraded. Since the radial velocity information in our low dispersion spectra is contained precisely in such underresolved features, a linear interpolation is unacceptable.

If an interpolation method is to avoid degrading the spectrum, it must have two characteristics. First, it must leave the information content of the spectrum unchanged in the following sense: if the entire stellar spectrum is shifted in wavelength by an arbitrary fraction of the sampling interval (which requires interpolation), the fast Fourier transform (FFT) power spectrum of the shifted stellar spectrum is identical to the power spectrum of the original stellar spectrum. Second, if the shifted stellar spectrum is shifted a second time in the same direction so that the sum of the two shifts is equal to an integer number of sampling intervals, the stellar spectrum after the second shift must be identical in form to the original stellar spectrum.

There is, for our spectra, an obvious interpolation method which has these essential characteristics. First, decide by what fraction of a sampling interval to shift the spectrum. Then, calculate the Fourier transform using the FFT. The shift of the stellar spectrum is equivalent to a phase shift of the Fourier transform, a different but calculable phase shift for each frequency of the transform. Thus, the next step is to shift the phases of the Fourier transform components. Finally, calculate the inverse FFT of the phase shifted transform. The result is the stellar spectrum shifted in wavelength. Although this interpolation method has the correct characteristics, for several reasons it is unsuitable for our needs as described. In order to change the nonlinear wavelength scale of our spectra to either a linear or logarithmic dispersion, the spectrum must be both shifted and stretched in wavelength. Because each sample point requires a slightly different wavelength shift of the spectrum, the interpolation method then requires a separate phase shift and inverse FFT for each sample point in the resultant spectrum. The amount of computer time required is devastating. In addition, the method must be used in full or not at all; there are no easily implemented half measures. Yet many spectra, particularly those spectra that are well resolved and have high signal-to-noise ratios, do not need the full power of the method.

We have elected to use an interpolated method which, although equivalent to the method just described, avoids its practical difficulties. Interpolation by introducing a phase shift in the Fourier transform is equivalent to interpolation in the original spectrum using a sinc function $S(x)$, where $S(x)=A \sin (\pi x) /(\pi x)$. Here, $x$ is a function of wavelength and is defined to change by $1.0 \mathrm{in}$ going from one sample point to the next (in our data $x$ is the diode number), and $A$ is the amplitude of the sinc function (see, for example, Jenkins and Watts 1968). Thus, to find the value of the stellar spectrum at some interpolation point $x^{\prime}$, fit the amplitude of $S\left(x-x^{\prime}\right)$ to the spectrum by least squares. The fitted amplitude is the correct value of the spectrum at $x^{\prime}$. This second method also has the two required characteristics of a good interpolation function, but like the first method, it also is impractical, in this case because the sinc function can be significantly different from zero even for very large values of $\left|x-x^{\prime}\right|$. Therefore, one is forced to truncate the sinc function at both ends at a finite value of $\left|x-x^{\prime}\right|$. In order to avoid edge effects, the truncation cannot be sharp, but must taper smoothly to zero. We have achieved good results by using a cosine function for the taper, and by tapering the final $10 \%$ at each end of the truncated sinc function. Our actual interpolation method then consists of these steps: (1) Choose a truncation length for the sinc function. For our data, we find that $\left|x-x^{\prime}\right| \lesssim 25-50$ diodes is appropriate. (2) Choose the point $x^{\prime}$ at which to interpolate the spectrum. (3) Evaluate $S\left(x-x^{\prime}\right)$ at the integer values of $x$ such that $\left|x-x^{\prime}\right|$ is less than the truncation length. Apply the taper to these values of $S\left(x-x^{\prime}\right)$, creating $S_{1}\left(x-x^{\prime}\right)$, the tapered sinc function evaluated at the sample points in the spectrum. (4) Take a weighted average of the spectrum sample points, with the weight of a sample point being given by the value of $S_{1}\left(x-x^{\prime}\right)$ at that point. The weighted average is the correct value of the interpolated spectrum at the point $x^{\prime}$. It can be shown easily that this weighted average gives the same value for the interpolated spectrum that is given by fitting $S_{1}\left(x-x^{\prime}\right)$ to the spectrum by least squares. (5) Should the spectrum have individual bad data points, the bad points are simply given zero weight in the weighted average. For interpolation near the ends of the observed spectrum, the non-existent data points off the edges of the spectrum are also given zero weight in the weighted average.

After the sinc function is truncated, our interpolation method does not have the two necessary characteristics specified at the beginning of this discussion, but it does approach the correct behavior in the limit at the length of the truncated sinc function is increased. In practice, large numbers of diodes need not be included in the weighted average. We have found that even when applied to a measured spectrum with a signal-to-noise ratio as poor as 10:1, the double-shift test retrieves the original spectrum, including the noise, with an accuracy of better than $1 \%$ using $\left|x-x^{\prime}\right| \lesssim 25-50$ diodes.

\section{REFERENCES}

Cowley, A. P. 1980, private communication. Griffin, R. F. 1967, Ap. J., 148, 465.

Heard, J. F. 1956, Pub. David Dunlap Obs., 2, 107.
Jenkins, G. M., and Watts, D. G. 1968, Spectral Analysis and Its Applications (San Francisco: Holden-Day).

Joy, A. H. 1956, Ap. J., 124, 317. 
Kiplinger, A. L. 1979, A.J., 84, 655.

Robinson, E. L. 1974, Ap. J., 193, 191

-. 1976, Ann. Rev. Astr. Ap., 14, 119. Smak, J. 1969, Acta Astr., 19, 287.
Tull, R. G., Vogt, S. S., and Kelton, P. W. 1979, S.P.I.E., 172, 90. Walker, M. F., and Chincarini, G. 1968, Ap. J., 159, 157.

Walker, M. F., and Reagan, G. H. 1971, I.B.V.S., No. 544

T. J. Montemayor, R. E. Nather, E. L. Robinson, and R. J. Stover: Department of Astronomy, University of Texas, Austin, TX 78712 